Social Justifications 1

Running Head: SOCIAL JUSTIFICATIONS FOR ANGER AND DISGUST

Social Justifications for Moral Emotions: When Reasons for Disgust Are Less Elaborated Than for Anger

\author{
Pascale S. Russell \\ Roger Giner-Sorolla \\ University of Kent
}

Author final version

Russell, P. S., \& Giner-Sorolla, R. (2011b). Social justifications for moral emotions: When reasons for disgust are less elaborated than for anger. Emotion, 11, 637-646. 


\begin{abstract}
In the present research we test the unreasoning disgust hypothesis: moral disgust, in particular in response to a violation of a bodily norm, is less likely than moral anger to be justified with cognitively elaborated reasons. In experiment 1, participants were asked to explain why they felt anger and disgust toward pedophiles. Participants were more likely to invoke elaborated reasons, versus merely evaluative responses, when explaining their anger, versus disgust. Experiment 2 used a between-participants design; participants explained why they felt either anger or disgust toward seven groups that either violated a sexual or non-sexual norm. Again, elaborated reasons were less prevalent when explaining their disgust versus anger, and in particular when explaining disgust toward a group that violated a sexual norm. Experiment 3 further established that these findings are due to a lower accessibility of elaborated reasons for bodily disgust, rather than inhibition in using them when provided. From these findings it can be concluded that communicating external reasons for moral disgust at bodily violations is made more difficult due to the unavailability of those reasons to people.
\end{abstract}

Keywords: Anger, Disgust, Reasoning, Moral Emotions 
Social Justifications for Moral Emotions: When Reasons for Disgust Are Less Elaborated Than for Anger

The reasons people give to justify their moral judgments have been studied in psychology ever since Kohlberg's ground-breaking work on moral reasoning (Kohlberg, 1969). Recent critiques of that work have highlighted the primacy of emotion and intuition in moral judgment (Haidt, 2001; Prinz, 2007), arguing that reasoning is a post-hoc social justification of moral judgments that mainly arrive quickly, with an emotional basis. For example, harm-based reasoning to justify condemning an act that explicitly harms no-one has been shown to have an emotional basis (Haidt \& Hersh, 2001), and is post-hoc, depending on more cognitive resources than does the moral judgment or its accompanying emotions (Gutierrez \& GinerSorolla, 2007). Even if seen as post-hoc, however, reasons are an interesting phenomenon within such views of moral judgment as Haidt's (2001) social intuitionist model, because they serve as a social output that reinforces and validates moral judgments.

In the research we report here, we compared the type of reasons that people give for feeling anger versus disgust towards groups that transgress moral values. Specifically, we propose that compared to anger, the related moral emotion of disgust is less likely to be justified using elaborated reasons: that is, references to the causes and consequences of the moral violation. By contrast, disgust should be equally or more likely to be justified with non-elaborated reasons, which are references to disgust itself, or to general evaluations of the moral violation. Our unreasoning disgust hypothesis further specifies that this difference should be most pronounced in situations that can give rise to bodily-moral disgust - that is, a moral violation that 
involves breaking a norm about the use of the body, such as a sexual violation. In other situations, such as when people report disgust toward groups that act unjustly without breaking a bodily norm, moral disgust will tend to be related more closely to anger, and will be justified with elaborated reasons in the same way as anger.

\section{Anger and Disgust}

Research on anger and disgust in moral contexts has mainly focused on determining the elicitors of these emotions. Moral anger is associated with concerns about harm and others' rights (Haidt, 2003), as well as with whether justice has been served (Goldberg, Lerner, \& Tetlock, 1999) and whether one has acted responsibly (Averill, 1983). While the elicitors of core disgust in non-moral situation appear to largely serve as cues to contagious disease; somewhat different elicitors of disgust apply in moral contexts (Marzillier \& Davey, 2004; Simpson, Carter, Anthony, \& Overton, 2006). Rozin, Haidt and McCauley (1993) argue that socio-moral disgust functions to preserve social order, and can be elicited by many different persons or groups, once they are seen to have done something morally wrong. Other emotion theorists argue that moral disgust is just extreme dislike (Taylor, 2007) or an emotion focusing on the badness of a person instead of an action (Ortony, Clore, \& Collins, 1990). However, other research finds that moral disgust is specifically elicited by violations of purity norms (Rozin, Lowery, Imada, \& Haidt, 1999), which primarily focus on the use of the body. As we will argue later, this distinction between bodily and non-bodily moral disgust is important for our predictions about elaborated reasoning.

In support of our hypothesis, disgust has been found to be a relatively inflexible emotion. As Rozin, Millman and Nemeroff (1986) observe, people maintain core disgust responses even when these are based on superficial, clearly non-rational 
perceptions. For example, after briefly touching a thoroughly disinfected cockroach to juice, longstanding negative beliefs about the juice persist (Hejmadi, Rozin, \& Siegal, 2004; Rozin et al., 1986). Disgust-related cues in social situations show a similar irrational biasing effect; for example, cues of bodily disability activate thoughts of disease and contagion, even when the disability is not due to disease (Park, Faulkner, \& Schaller, 2003).

Moving to the realm of moral judgments, research in support of Haidt's (2001) moral intuitionist position has found a moral dumbfounding effect that seems to reflect the irrational nature of disgust responses. In moral dumbfounding, people often fail to come up with elaborated reasons for strongly and quickly expressed moral judgments. More commonly, they give re-statements of their emotions or their judgment, such as “It’s disgusting” or “It’s just wrong” (Bjorklund, Haidt \& Murphy, 2000). These researchers presented participants with morally distasteful acts in scenarios ruling out likely justifications for negative judgments (for example, brothersister incest in which nobody gets hurt, nobody finds out, and there is complete consent). Even though this research did not assess anger and disgust specifically, it is suggestive that moral dumbfounding was most strongly shown among scenarios violating norms about the bodily matters of sex and eating, such as the incest taboo example. A scenario in which participants symbolically sold their soul, for example, did not produce dumbfounding effects as strongly.

Likewise, Haidt and Hersh (2001) have found that persons - especially political conservatives -- often could not explain their condemnation of consensual sexual behaviors, responding instead with evaluative statements, such as "It is just wrong.” In that study, emotions rather than reasons predicted moral judgment. Although the research did not distinguish between specific emotions, it is also 
suggestive that these "dumbfounding” like responses were found in moral judgments of sexual norm violations, which are likely to elicit bodily moral disgust.

Anger, compared to disgust, appears to be a more flexible emotion and is often influenced by abstract cues in the situation. For example, Goldberg, Lerner and Tetlock (1999) found that once participants learned that justice had been served, this decreased anger and no longer influenced future judgments. Research has also found that the anger experience is influenced by one's relationship to the target (e.g., Fischer \& Roseman, 2007; Weber, 2004), and that anger is influenced by abstract contextual cues, such as harm and intentionality, while disgust only responds categorically to whether or not a bodily norm violation has occurred (Russell \& Giner-Sorolla, 2009; see also Gutierrez \& Giner-Sorolla, 2007). Thus, there are many factors that appear to modify anger, while disgust is more resistant to changes in context. This suggests that the reasons for feeling moral anger may be more open for discussion in everyday life, and that people gain more practice in justifying their anger with reasons. On the other hand, when someone feels moral disgust, the rules about the body that elicited it may not be as open for debate in the person's culture; disgust feelings about bodily violations tend to be shared within a culture, not argued about.

There appears to be a functional difference between anger and disgust as well. Disgust is defensive (Izard, 1977), encouraging the individual to turn attention elsewhere and to avoid the situation, with few immediate social repercussions. However, anger has been described as an emotion that creates social accountability (Averill, 1983), which often leads the angry individual to think about how his or her response will impact others. Because anger encourages aggressive approach behaviors at least in the short term (Fisher \& Roseman, 2007) and often leads to more risky decision-making (Lerner \& Keltner, 2001) anger can be costly to the individual 
and to society if it is not justified. It may thus be functional to consider why one feels anger in the first place, and to justify one's anger to others with appropriate reasons. Bodily and Non-Bodily Disgust

Our hypothesis carries an important qualification, distinguishing between forms of disgust involving the violation of bodily moral norms and those that involve the violation of non-bodily moral norms. In the first category we place societal taboos about the use of the body, such as norms against cannibalism, incest, or eating meat from forbidden animals. The elicitors of bodily moral disgust are learned as inflexible rules or "shalt nots" and are generally not open to controversy in a homogeneous society. These features may explain why persons are not motivated to give elaborated reasons for their bodily moral disgust: they normally do not need to provide a justification for this emotion, and considerations of intent and consequences do not affect whether or not someone has violated the sexual or dietary norm.

Non-bodily moral disgust, on the other hand, is elicited when someone is seen as acting in a morally heinous manner that does not involve norms about the body, such as betraying, exploiting, or being bigoted toward someone. These are more complex judgments about human behavior involving attributions and theory of mind. Non-bodily moral disgust has been shown, unlike core disgust, to share a good deal of variance with anger (Simpson, Carter, Anthony, \& Overton, 2006). Unlike bodily moral disgust, even members of a homogeneous society can disagree over whether a given action should evoke non-bodily moral disgust, similar to disagreements about anger. For these reasons, we expect moral disgust without any bodily element to show a similar pattern of reasoning to moral anger. 


\section{Overview of present research}

Based on the above considerations, we expect bodily moral disgust, as opposed to moral anger and non-bodily moral disgust, to be justified principally with non-elaborated reasons, which are evaluative or emotional in nature, rather than with elaborated reasons, which are externally grounded arguments. Non-elaborated reasons reiterate subjective feelings but ignore the causes and consequences of the group or its behavior; for example, “I don’t like them” or “They are disgusting.” Elaborated reasons, however, justify feelings with external concepts beyond mere evaluation; for example, "They make me feel angry because they abuse the power they have been given”. We expect more elaborated reasons to be given when participants justify their anger and non-bodily disgust.

In our first two experiments, participants expressed their own reasons for feeling anger and disgust, toward various social groups. These free responses were then content-coded for whether they represented elaborated or non-elaborated reasons. Terms for disgust and anger are sometimes confused within the English language (Nabi, 2002; Russell \& Fehr, 1994); therefore, both the emotion term and the corresponding facial expression were presented to illustrate each emotion, clarifying what emotion participants were meant to explain. This technique has worked to reduce correlations between ratings of anger and disgust in similar moral contexts (Gutierrez \& Giner-Sorolla, 2007). The third experiment presented similar emotion cues; however, we manipulated whether or not emotion explanations were provided when individuals were justifying their emotions, to see whether or not participants continued to prefer less elaborated reasons for disgust when elaborated reasons appropriate to disgust were made available to them. 


\section{Experiment 1}

This experiment looked at anger and disgust toward the group of pedophiles. This group was chosen because it evokes the moral emotions anger, disgust, contempt and fear (Marzillier \& Davey, 2004) and because it violates a sexual, bodily norm. Two other emotions were included primarily for comparison purposes: contempt, as the third member of the other-condemning triad of emotions (Haidt, 2003) and fear, as a negative other-centered emotion that is not primarily moral in nature. The primary focus, however, was on the comparison between anger and disgust.

\section{Method}

\section{Participants}

This study recruited 52 participants (12 males, 39 females and 1 who did not complete the gender item) between the ages of 18 to $58(M=23.02, S D=7.37)$, all students at the University of Kent. The incentive for participating was entry into a raffle to win a $£ 50$ cash prize.

\section{Materials and Procedure}

Individuals were first given a brief definition of pedophilia, and then completed the questionnaire. The questionnaire's four sections each presented one of the four emotions- anger, disgust, contempt and fear- with a picture of a woman displaying the appropriate emotional facial expression, taken from Tracy \& Robins, (2008), and the emotion's name as a label. Participants were asked whether they experienced the emotion toward pedophiles (yes or no) and to write down the reasons why they felt each emotion. They were told to give reasons for each emotion in particular, as distinct from other emotions; that their responses could be detailed or as basic as they wished; and that they could write down as many or as few of them as they wish. The emotions were presented in a counterbalanced order. 


\section{Coding Scheme}

When participants wrote multiple judgments in a single response, usually by using multiple sentences, these were split into separate statements. Three independent graduate student coders then rated each participant's statements separately. Coders were native English speakers who were not aware of our hypothesis. Statements were coded as a 1 if they contained an elaborated reason or 0 if they contained a nonelaborated reason. An elaborated reason was defined as an objectively rather than subjectively argued justification. Coders were told that merely giving an overall evaluation or a subjective feeling as a reason did not count as an appropriate reason; elaborated reasons had to refer to the causes and/or consequences of the group or its behavior. For example, the statement “pedophiles violate others’ human rights”, would count as an elaborated reason. On the other hand, a statement was coded as a non-elaborated reason if a participant merely stated a subjective feeling or evaluation: for example, "pedophiles are bad people.” The coders had good agreement, with an intraclass correlation of .81.

From the coding, four variables were created for each of the four emotions for each participant. First, the total amount of statements given was counted. The elaborated reasons variable was based on the mean of all three coders’ judgments about the number of elaborated reasons. The non-elaborated reasons variable was calculated by subtracting the amount of elaborated reasons from the total statements given. Additionally, a non-elaborated reason could further be judged as tautological if it referred only to a literal synonym of the emotion requested (e.g., "Because they are gross” as a reason for disgust). This dichotomous variable was assessed by a single coder, using Johnson-Laird and Oatley's (1989) emotion lexicon as an a priori way to determine emotion synonyms. 


\section{Results}

\section{Identifying Emotions}

In six cases (participant-emotion combinations), the written responses either explicitly indicated a different emotion than asked for, or denied feeling the emotion despite having indicated "yes" in the yes/no emotion question. These cases, in addition to all "no" responses to feeling the emotion, were excluded from the final data set. This left us with 42 cases for disgust, 35 cases for anger, 14 cases for fear, and 27 cases for contempt.

\section{Emotion Explanations}

Elaborated reasons and non-elaborated reasons were both analyzed using a mixed model procedure in SPSS version 16, with emotion as a fixed factor and participant as a random factor. The mixed model procedure was chosen due to the existence of missing data from participants who did not identify particular emotions (Kenny, Kashy \& Bolger, 1998).

For elaborated reasons there was a main effect of emotion, $F(3,114)=4.65, p$ $=.004$, indicating a significant difference between the number of elaborated reasons for the different emotions (see Figure 1 for means). Post hoc comparisons using the Dunn-Sidak correction revealed that anger elicited more elaborated reasons than disgust did (adjusted $p=.005$ ), and a trend toward more elaborated reasons for fear than disgust (adjusted $p=.09$ ), no other comparisons were near significant. The previous analysis was then repeated on the amount of non-elaborated reasons, which also indicated a significant main effect for emotion, $F(3,114)=5.52, p=.001$ (see Figure 1 for means). The post hoc comparison between non-elaborated reasons for disgust and anger was also significant using the Dunn-Sidak correction (adjusted 
$p=.001$ ), and showed the converse effect, with more non-elaborated reasons for disgust than anger. No other comparisons were significant.

To increase the validity of the comparison between anger and disgust, a paired samples t-test was carried out upon only those 29 participants who reported feeling both those emotions. Even among this subsample, people used more elaborated reasons to explain their anger than their disgust, $t(27)=3.63, d=0.86, p=.001$ (Disgust: $M=0.38, S D=0.40$; Anger: $M=0.71, S D=0.37$ )

Finally, ten participants gave a tautological response explaining their disgust, while only one participant gave a tautological response for anger; this was significantly different from an equal distribution of tautologies between the two conditions, $\chi^{2}(1, \mathrm{~N}=22)=5.87, p=.02$. Neither fear nor contempt elicited tautological statements.

\section{Experiment 2}

When judging a group that could be expected to raise bodily-moral concerns, participants within Experiment 1 were less likely to give elaborated reasons when explaining their disgust than when explaining their anger. However, the next experiment explicitly tested a further prediction of the unreasoning disgust hypothesis: that this difference in the quality of reasoning would not be seen when explaining disgust in reaction to non-bodily moral violations. Also, because participants within the previous experiment could compare their reasons for anger and for disgust in a within-participants design, it was important to vary emotion between participants, making the difference between the two emotions less obvious. Finally, this experiment sought to generalize its findings by increasing the number of groups under consideration, with multiple groups representing both bodily and non-bodily moral violations. 
Anger and disgust alone were manipulated between participants in this experiment. Each participant evaluated multiple groups, each of which violated either a bodily or non-bodily moral norm, varying within participants. We predicted that participants would be least likely to offer elaborated reasons when explaining disgust felt toward bodily groups, compared both to non-bodily groups and to explanations of anger. The current experiment also controlled for the possibility that more elaborated reasons were given for anger simply because it was experienced more intensely than disgust. Now, emotion intensity was assessed on a continuous scale, as well as by a dichotomous yes or no, so that it could be used as a covariate.

\section{Method}

\section{Participants}

This study recruited 70 students at the University of Kent in the same way as in Experiment 1, 20 men and 50 women between the ages of 18 and 43 ( $M=21.11$, $S D=4.99)$. None of them had participated in the prior experiment.

\section{Materials and Procedure}

The first page of the questionnaire used the same instructions, pictures and words as Experiment 1. Participants were randomly assigned to the anger or disgust condition. Each page of the questionnaire presented the emotion face from the instruction sheet, gave the name of one of the seven groups, and asked for a yes/no rating and a scaled rating of their intensity of that emotion toward the group (9 point scale from 1: “Not at all” to 9: “Completely”). After rating the emotion, individuals were asked to give reasons for experiencing this emotion, separately from any other emotions felt. The seven groups used were expected to elicit greater or lesser degrees of moral condemnation from at least some of our participants. Four of these groups were chosen because they may violate a sexual bodily norm (prostitutes, 
homosexuals, voyeurists, necrophiliacs; these last two groups were briefly defined to participants), while the other three groups were chosen because they may violate nonbodily norms (activist feminists, Islamic religious fundamentalists, crooked politicians).

\section{Coding Scheme}

Two of the three independent coders involved in Experiment 1 rated responses using the same coding scheme as in that experiment on a subsample of the data set (150 statements). The main variable, elaborated reasons code, was found to be reliable within this study, intraclass correlation $=.79$. One of the coders then rated the full data set.

\section{Results}

\section{Identifying Emotions}

Each response toward a specific group in which the participant did not report feeling the emotion in question (anger or disgust) was excluded from analysis, on the same grounds as in Experiment 1. The final data set consisted of 253 responses, with 125 responses (72 bodily; 53 non-bodily) in the disgust condition, and 128 responses (59 bodily; 69 for non-bodily) in the anger condition, emotion intensities for these responses are displayed in Table 1. This pattern of yes/no responses also showed that people tended to report disgust proportionally more than anger toward bodily versus non-bodily groups, $\chi^{2}(1, \mathrm{~N}=253)=7.18, p<.01$; however, this difference was not large, phi $=.17$, and left an adequate number of cases in all cells. Simple effects analyses also indicated a significant difference on the continuous measure of disgust for the bodily and non-bodily groups, $F(1,249)=4.18, p=.04$; people were more likely to feel disgust toward bodily norm violators than non-bodily norm violators. On the other hand, there was no difference on the anger measure for the group types, $F$ 
$(1,249)=0.03, p=.87$. Also, there was no significant relationship between emotion intensity and elaborated reasons, $r(253)=-0.05, p=.46$, thus, it was not necessary to control for emotion intensity as a confound that could affect elaborated reasoning.

\section{Emotion Explanations}

Mixed model analysis was again used, including a random term for the participant, and fixed terms for the effects of theoretical interest: main effects of emotion (anger vs. disgust), group type (bodily vs. non-bodily), and specific group nested within group type. The model also included the interactions Emotion x Group Type, and Emotion x Specific Group.

For the amount of elaborated reasons there was a significant main effect for emotion, $F(1,239)=8.45, \gamma=.07, p=.004$, such that anger elicited more elaborated reasons (adj. $M=0.95$ ) than did disgust (adj. $M=0.69$ ). The type of group also had a main effect, $F(1,239)=4.88, \gamma=.39, p<.05$, such that bodily norm violators elicited fewer elaborated reasons (adj. $M=0.74$ ) than did non-bodily norm violators (adj. $M=$ 0.92). As expected, the interaction between these two factors was also significant, $F$ $(1,239)=4.23, \gamma=.37, p=.04$ (adjusted means displayed in Figure 2). A simple effects breakdown showed that for bodily norm violators, anger elicited more elaborated reasons than disgust, $F(1,239)=10.81, p=.001$. For non-bodily norm violators, there was no emotion effect, $F(1,239)=0.36, p=.55$; anger and disgust had relatively equal and high levels of elaborated reasoning. Neither the nested main effect of the specific group nor the interaction of that effect with emotion condition was significant, both $p>$.36. This shows that effects generalized across groups within each category. Analyses of non-elaborated reasons revealed a similar pattern of effects in the opposite direction. 
Statements were again coded for tautologies. No tautological answers were used to justify anger; however, 14 disgust responses were tautological, 12 for bodily groups and 2 for non-bodily groups. This difference between group types within disgust was different from a proportionally equal distribution of tautologies within disgust, $\chi^{2}(1, \mathrm{~N}=28)=4.53, p<.05$. Thus, only for disgust did people offer the emotion itself as a justification, and they were most likely to do so when explaining disgust toward groups that might violate a bodily norm.

\section{Discussion}

Our results so far support the unreasoning disgust hypothesis, in that anger was more likely to elicit elaborated reasons, compared to bodily moral disgust. These effects were found using a variety of social groups as targets and two different experimental designs. Moral disgust at groups whose violation was non-bodily, however, was more similar to anger than to bodily disgust in the relatively high proportion of elaborated reasons elicited. These findings, and the relatively high levels of reasoning exhibited by the comparison emotions in Experiment 1, suggest that there is something special about bodily moral disgust that leads to a lower level of elaborated reasons.

\section{Experiment 3}

The next experiment examined possible explanations for unreasoning disgust. One possibility is that the kind of things that elicit bodily-moral disgust just do not lend themselves to elaborated reasoning, so that we were asking the impossible of our participants. However, scholars have found a number of reasons for bodily-moral disgust, many of which would be plausible to a lay person. For example, in various contexts, disgust has been shown to arise out of concern with contamination and impurity (Curtis \& Biran, 2001; Rozin et al., 1999); perception of a threat to values 
(Cottrell \& Neuberg, 2005; Olatunji, 2008); violation of a social taboo (Gutierrez \& Giner-Sorolla, 2007); or abnormal uses of the body (Giner-Sorolla, Bosson, Caswell, \& Hettinger, 2009). Any of these explanations for bodily disgust, if given by our participants in the first two studies, would have been scored as elaborated reasons. Therefore, it is not likely that our results simply reflect the impossibility of creating reasons relevant to disgust.

A more likely possibility is that our participants were less knowledgeable about elaborated disgust reasons, making those reasons less available or accessible. This might come about because we are less used to having to justify bodily disgust than anger. Because anger and non-bodily disgust involves conflict between the rights of individuals within the culture, the expression of these emotions may be more frequently challenged among peers, creating disagreements that need to be resolved by discussion. Social peers in the same culture may just take for granted that the same sexual and dietary violations are disgusting. Therefore, people may be less knowledgeable of the elaborated reasons that can be used for bodily disgust. This suggests that if we made available elaborated reasons that were specific to moral disgust, people would use them, and the anger-disgust difference in elaborated reasons would disappear.

Opposing our hypothesis would be the alternate explanation that elaborated reasons for bodily disgust are equally accessible, but just not reported by participants. That is, people may know why they feel disgusted, but feel inhibited to give external explanations for disgust versus anger, so that even if elaborated reasons specific to bodily-moral disgust were made available, people would not use them. The next experiment tests this alternative against our availability hypothesis -- that if plausible 
elaborated reasons for disgust were made available to people, they would be used to justify feelings of disgust.

In Experiment 3, we manipulated whether or not emotion explanations were provided when participants were justifying anger or disgust. The statements that were provided included both elaborated and non-elaborated reasons for both emotions. If bodily disgust evokes fewer elaborated reasons because the reasons are simply not available, making such reasons available should eliminate the advantage that anger has over disgust. Further improving our design, group type as well as emotion were now both treated as between participants variables, so that participants could not compare the reasons that they were giving for the two group types or for the emotions. We also created a tighter manipulation of the type of norm violation by describing two social groups that were identical except for the bodily or non-bodily nature of their violation, reducing the possibility that other differences between groups could account for the effects.

\section{Pretest}

In order to obtain a list of statements that could be used for this experiment, 10 psychology postgraduate students (five males, five females) between the ages of 22 and $32(M=26, S D=3.33)$ rated 44 statements in a pretest. The rating used a single item complexity scale from 1: Not at all complex to 9: Extremely complex. Instructions for the pretest are included in Appendix A.

Some statements were taken from participant-provided answers in the first two studies, and others were created by the experimenters. The aim was to include an equal number of statements appropriate to each emotion that would be coded as nonelaborated reasons and elaborated reasons according to our previous scheme. Elaborated reasons were of two kinds. Impurity/abnormality reasons were statements, 
theoretically related to moral disgust, and referring to how the group is abnormal, of inferior character, or impure. For example, the statement "They violate socially imposed boundaries” was considered to be an elaborated reason that was appropriate for disgust. On the other hand, harm/rights reasons were statements, theoretically related to moral anger, referring to how the group causes harm, injustice, or violation of people’s rights (e.g., “Their actions violate others’ basic human rights”). Nonelaborated reasons were either general evaluative statements (e.g., “I don’t like them”) or tautologically referred to the emotion itself (e.g., “They are disgusting”).

Based on the pretest, statements were chosen so that within each reason type (non-elaborated reason versus elaborated reason) the reasons related to anger and to disgust were about equally complex, with an equal amount of statements for each reason type. So, the list of elaborated reasons involved 8 reasons of the harm/rights type (theoretically leading to anger) and 8 of the impurity/abnormality type (theoretically leading to disgust) which were of comparably high complexity. (Impurity/abnormality: $M=3.31$, Harm/rights: $M=3.40$ ). The non-elaborated reasons had lower rated complexity, and included five statements directly referring to disgust and its synonyms $(M=1.74)$ and four statements referring to anger and its synonyms $(M=2.05)$. There were also five general statements which were evaluative in nature, having on average a lower complexity score $(M=2.40)$.

The total numbers of elaborated and non-elaborated reasons were calculated for each participant, including emotion specific as well as general statements. We also calculated each participant's total number of each kind of emotion-specific elaborated reason (impurity/abnormality and harm/rights).

A second goal of the pretest was to select the target groups for this experiment. We askedfor participants to rate six hypothetical groups on the following 
variables: disapproval, human rights violation, bodily norm violation, social norm violation and wrongness. All measures were 9 point scales that ranged from 1: Not at all to 9: Completely. Two of the six groups were chosen because they were closely matched on overall disapproval (mean value of disapproval and wrongness for each group was calculated), $t(9)=0.35, d=0.09, p=.74$. However, one scored high on bodily norm violation, while the other did not, $t(9)=3.73, d=1.39, p=.005$. The bodily norm violating group was described as people who like to rub their genitals on their co-workers as they walk past them. The non-bodily norm violating group was described as people who like to send death threats to their co-workers anonymously because they feel it adds excitement to their job. For all other measures there was no significant difference between the two selected groups, all $p<.45$. The descriptions of the two groups that were used were identical except where the moral violation was mentioned. One of the groups can be seen as violating a bodily norm because the group members like to rub their genitals on their co-workers as they walk past them. The other group can be perceived as violating a social norm because the group members like to send death threats to their co-workers anonymously because they feet it adds excitement to their job.

\section{Method}

\section{Participants and Research Design}

There were 155 participants (39 males, 117 females and 2 who did not identify their sex) between the ages of 17 and $43(M=20.85, S D=4.27)$. Participants were given either $£ 3$ or partial course credit. The design of this study was a 2 (Explanations: provided or not provided) x 2 (Emotion: anger or disgust) x 2 (Group type: bodily vs. non-bodily violation) between participants design. 
Participants were randomly assigned to either the "explanations provided” or “explanations not provided” condition. In the first condition, participants were given the full list of statements generated through pre-testing, and asked to build an emotion explanation with the statements in order to explain the anger or disgust they might feel toward one of the groups (bodily or non-bodily). On the other hand, participants within the explanations not provided conditions were asked to give a list of statements that they would use to explain their anger/disgust toward one of the groups (bodily or non-bodily). Before these specific instructions, both the emotion word and face were presented as a cue to what emotion participants were to explain within the general instructions, using the same stimuli as the previously conducted experiments. The general instructions also emphasized that they should explain their emotion as they would to other persons (instructions are included in Appendix B).

Participants then rated how much they felt the following emotions toward the group: sickened, outraged, moral disgust, inspired, sympathy, infuriated, proud, physical disgust, hatred, contempt, angry and afraid, on a 7 point scale $(0=$ Not at all to 6 = Extremely). The disgust words (sickened, moral disgust and physical disgust) were found to be a reliable scale (Cronbach $\alpha=.78$ ). The anger words (outraged, infuriated, angry) were also found to be a reliable scale (Cronbach $\alpha=.87$ ). Overall anger and disgust intensity variables were calculated as the mean of the appropriate emotion words. We created an "appropriate emotion intensity” variable, which was the mean of disgust words for people within the disgust conditions and the mean of anger words for people within the anger conditions. Again, this variable was not significantly correlated with the amount of elaborated reasons, $r(155)=-0.02, p=.77$, thus, it was not necessary to control for this variable within the main analyses.

\section{Coding Scheme}


Participants’ free responses from the explanations not provided conditions were coded by the first author using the same coding scheme as the previous experiments, because the coding scheme had achieved reliability across the two previous experiments. Coding was blind to condition.

\section{Results}

\section{Emotion Explanations}

To test the availability hypothesis, specific contrasts of theoretical interest were carried out on the total number of elaborated reasons (see Figure 3 for means). Within each explanations condition (provided versus not provided), simple main effect and interaction contrasts were carried out for the Group Type x Emotion design. This revealed no significant effects in the "explanations provided" condition (all $p>$ .12), but a significant main effect of Emotion in the "explanations not provided" condition, $F(1,147)=8.07, p<.01$, which was qualified by an Emotion $x$ Group Type interaction, $F(1,147)=4.16, p<.05$. A similar analysis on non-elaborated reasons showed no significant effects in either explanation condition, all $p>.20$.

We further carried out planned comparisons between the disgust and anger conditions, within each of the four combinations of Explanation x Group Type. As expected, the only significant contrast was the comparison between bodily disgust and bodily anger when emotion explanations were not provided, $F(1,147)=12.40, p<.01$. All other planned contrasts were nonsignificant with $\mathrm{F}<1$.

To sum up, when the experiment made elaborated reasons available, this eliminated the asymmetry in reasoning between anger and disgust that had been found for bodily-moral violations within the previous reason experiments. On the other hand, when elaborated reasons were not available, the results of the previous studies 
were replicated; participants still gave fewer elaborated reasons for disgust versus anger towards a group violating a bodily norm.

\section{Type of Elaborated Reasons}

Further analyses were carried out in order to see what kind of elaborated reasons were selected within the "explanations provided" condition. Mixed model GLM analysis was carried out using reason type (impurity/abnormality elaborated reasons versus harm/rights elaborated reasons) as a within participants variable. Emotion (disgust versus anger) and Group type (bodily versus non-bodily) were entered as between participants variables in this analysis. The amount of elaborated reasons was used as the dependent variable.

There was a significant main effect for reason type, $F(1,72)=112.76, p<.001$, partial $\eta^{2}=.61$, such that across the conditions participants were more likely to give harm/rights elaborated reasons (adj. $M=2.17$ ) than impurity/abnormality elaborated reasons (adj. $M=0.54$ ). A significant interaction between reason type and group type was also observed, $F(1,72)=10.49, p=.002$, partial $\eta^{2}=.13$. Simple effects analyses indicated that more impurity/abnormality elaborated reasons were elicited by the bodily group than the non-bodily group, $F(1,72)=6.20, p=.02$, partial $\eta^{2}=.08$. On the other hand, the non-bodily group generated more harm/rights elaborated reasons than the bodily group, $F(1,72)=6.98, p=.01$, partial $\eta^{2}=.09$.

The three-way interaction between emotion, reason type and group type was found to be significant, $F(1,72)=4.88, p<.05$, partial $\eta^{2}=.06$ (means displayed in Figure 4). Simple effects analyses revealed that more impurity/abnormality elaborated reasons were selected within the bodily disgust condition than within the non-bodily disgust condition, $F(1,72)=14.09, p<.001$, partial $\eta^{2}=.16$. Conversely, more harm/rights elaborated reasons were selected within the non-bodily disgust condition 
than within the bodily disgust condition, $F(1,72)=8.23, p=.005$, partial $\eta^{2}=.10$. However, the anger conditions did not differ in the type of elaborated reasons that were selected.

\section{Discussion}

Experiment 3 supported the availability hypothesis. Making elaborated reasons available led to equal selection of elaborated reasons to justify disgust and anger, whereas when explanations were not made available, disgust at bodily violations was again explained with fewer elaborated reasons than other moral emotions and contexts. This indicates that while persons normally find it difficult to access or generate elaborated reasons for disgust, they will readily use them if they are provided.

The analysis of the types of reasons selected when explanations were provided sheds further light on these results. Participants were not indiscriminately picking elaborated reasons; instead, they selected impurity/abnormality reasons more often to explain their disgust at a bodily violation than disgust at a non-bodily violation, or anger at any violation. Thus, the lesser use of elaborated reasons to explain bodily disgust in our first two studies, and in the "explanations not provided" condition of this study, can be balanced out by a greater use of elaborated impurity/elaborated reasons, but only when these were made available.

\section{General Discussion}

These findings provide evidence for our unreasoning disgust hypothesis. Across three experiments using different methods and different social targets, more non-fewer elaborated reasons were used to justify one's bodily-moral disgust toward social groups, as opposed to anger or non-bodily disgust. We also found that this effect is-was driven by a lesser accessibility of elaborated reasons appropriate for 
disgust, not by reluctance to use such reasons when provided, or by the impossibility of generating elaborated reasons for disgust. Our experimental designs became progressively more controlled, replacing within-participants with betweenparticipants manipulations, in order to ensure that participants could not compare the type of reasons that they were giving. We also used a variety of social groups as targets, and in our final experiment we ensured that our groups only differed by the type of moral norm that they violated. Also, in each of our studies, the intensity of the emotion felt had little to do with the number of elaborated reasons generated,-. making itThus, it is notim_plausible that our findings are-were merely due to different intensities of anger versus disgust.

Cumulatively, the results of these experiments suggest that there is something unique about bodily moral disgust, requiring a distinct type of justification. The kind of broadly evaluative responses ("Because they are bad") and tautological statements (“Because they are disgusting”) used to justify disgust toward sex offenders, prostitutes, and other bodily norm violators appear to be the best effort of participants to provide an explanation for their moral disapproval.

These studies support differences that have been found between two different contexts of disgust, involving violations of bodily versus non-bodily norms. Because our studies used both facial expressions and words to describe disgust and anger, the results involve broader understanding of these emotions than just the Englishlanguage term "disgust," which arguably can be used to refer to the basic emotion of anger. All the same, the similarities we found in the amount and type of elaborated reasons between anger and non-bodily moral disgust support a stronger affinity between these two emotional contexts than between bodily moral disgust and anger, 
as does the research of Simpson et al. (2006), which found stronger correlations between non-bodily moral disgust and anger than between bodily disgust and anger.

On the basis of our findings, we can also conclude that groups that are viewed with bodily disgust, as opposed to anger, will show different ways of establishing and ultimately changing prejudice towards-against them. Extensions of prejudice theory into the realm of intergroup emotions have emphasized the ability of specific negative emotions to predict different prejudicial action tendencies (Mackie \& Smith, 2002). These results add to the usefulness of intergroup emotion theory by showing that different negative emotions also carry different cognitive consequences for justifying prejudice against outgroups. Prejudice against groups such as gay men predominantly involves disgust (e.g., Cottrell \& Neuberg, 2005; Olatunji, 2008).

Our results further suggest that disgust-based prejudice is not likely to arise just through reasoning or discussion of threats sexual minorities may pose, realistic or otherwise. Since persons are less able to articulate the causes of bodily moral disgust, this implies that reasoned forms of persuasion relying on cognitive responses (Greenwald, 1968) may have more impact on anger-based and fear-based attitudes than on disgust-based attitudes. Simple social learning by association ("they are just disgusting”) may be a better model for the acquisition of anti-gay attitudes. Furthermore, if disgust-based attitudes are not justified by elaborated reasoning, it is unlikely that they can be changed by elaborated reasoning. Exposure to positive examples of sexual minorities, or to heterosexual peers with tolerant and approving attitudes toward gay men and lesbians, may thus be a more effective way of fighting homophobia.

In the philosophy of law, our findings support the emotion theory of Martha Nussbaum (2004); in particular her argument that anger, unlike disgust, “rests on 
reasoning that can be publicly articulated and publicly shaped” (p.99). Rather than articulating reasons, Nussbaum argues, the best way to persuade someone to feel disgusted is to describe concrete disgusting qualities, such as ooziness or stickiness. Our studies add to this insight by showing that, when disgusting qualities are moral instead of material, people often describe only the fact of their emotion or their evaluative feeling when justifying bodily disgust, apparently hoping to convince other people more by social contagion than by argumentation. Nussbaum gives another reason why disgust may be less elaborated: the primary elicitors of disgust are often shared among members of a given society. Thus, bodily disgust may be an unreasoned emotion because its reasons are not often socially asked for, making it more likely that persons will fail to reason about disgust when asked to do so. It is only when elaborated reasons are made available that we saw people using this type of reasons to justify bodily disgust. Although elaborated reasons for bodily disgust do exist and are recognized, they may be harder to retrieve because they are not normally used in social discourse.

This research also gives initial empirical evidence for the role that these two emotions should have in legal judgments. Supported by her theorizing on emotion, Nussbaum (2004) claims that it is more unreasonable for disgust, versus anger, to influence judgments in a liberal system of law and justice. This research then supports this view by indicating that anger more so than disgust is an emotion that elicits elaborated reasoning, which encourages an assessment of the current situation. On the other hand, disgust does not promote individuals to consider whether or not their disgust is warranted, perhaps because they generally assume that others agree with their response. Crucially, the kind of reasoning we have shown to accompany disgust is unwarranted. In conclusion, persons involved in legal decisions, and the 
general public as well should take into account this difference between anger and disgust. This consideration will then hopefully lead persons to question whether or not their disgust is warranted, and if it is necessary to modify their feelings of disgust.

Finally, these results support and clarify the mechanisms of Haidt's social intuitionist model of moral judgment (2001). Although Haidt's model is often represented as an argument for the primacy of emotion in moral judgment, it also gives an important role to post-hoc reasoning in the social validation and transmission of moral responses. Our results qualify this role, by indicating that the moral disapproval emotions of anger and disgust are typically associated with different types of social justifications. While elaborated argumentation is used to support and justify anger, a simpler kind of reasoning that depends on restatement of the emotion or of basic evaluations of the transgressor is relied on to support and justify bodily disgust at a moral violation. Our findings also suggests a boundary condition on the findings of moral dumbfounding that have supported the primacy of emotion in moral judgment. Dumbfounding, or the inability to give any reason for a moral response beyond tautologies, will be most likely to arise when justifying moral disgust at scenarios such as consensual incest. Our results suggest that even though other moral emotions can come before their justifications, people will be likely to give copious reasons when justifying, for example, moral anger or disgust at corrupt investment bankers.

It may be that elaborated reasons are normally not necessary to justify bodily disgust because the mere fact that a socially valued person feels disgusted is enough to elicit disgust. In contrast, because people are aware that anger should be socially justified, they may require more reasons to ultimately justify an empathetic response to an angry friend. In fact, we suspect that our findings could be supported from the 
demand as well as the supply side; that is, people might be more likely to accept and support another person's moral anger when it is expressed using appropriate reasons, whereas reasoning would make less of a difference for moral disgust. As socially embedded constructs, moral emotions and their justifications may ultimately be best studied as dynamic interactions between individuals; our present research looking at one side of the equation presents some intriguing possibilities about why communications of anger and disgust between individuals show different amounts of cognitive elaboration.

\section{References}

Averill, J. (1983). Studies on anger and aggression: Implications for theories of emotion. American Psychologist, 38, 1145-1160.

Bjorklund, F., Haidt, J., \& Murphy, S. (2000). Moral dumbfounding: When intuition finds no reason. Lund Psychological Reports, 2, 1-23.

Cottrell, C. A., \& Neuberg, S.L. (2005). Different emotional reactions to different groups: A sociofunctional threat-based approach to prejudice. Journal of Personality and Social Psychology, 88, 770-789.

Curtis, V., \& Biran, A. (2001). Dirt, disgust, and disease - is hygiene in our genes? Perspectives in Biology and Medicine, 44, 17-31.

Ekman, P. (1999). Basic emotions. In T. Dalgleish \& M. Power (Eds.), Handbook of cognition and emotion (45-60). New York: John Wiley \& Sons. 
Fisher, A.H. \& Roseman, I.J. (2007). Beat them or ban them: The characteristics and social functions of anger and contempt. Journal of Personality and Social Psychology, 93, 103-115.

Giner-Sorolla, R. Bosson, J., Caswell, T.A. \& Hettinger, V.E. (2009). Emotions in sexual morality: Testing the separate elicitors of anger and disgust. Unpublished Manuscript.

Goldberg, J.H., Lerner, J.S., \& Tetlock, P.E. (1999). Rage and reason: the psychology of the intuitive prosecutor. European Journal of Social Psychology, 29, 781795.

Greenwald, A. G. (1968). Cognitive learning, cognitive response to persuasion, and attitude change. In A. G. Greenwald, T. Brock, \& T. Ostrom (Eds.), Psychological foundations of attitudes. New York: Academic Press.

Gutierrez, R. \& Giner-Sorolla, R. (2007). Anger, disgust, and presumption of harm as reactions to taboo-breaking behaviors. Emotion, 74, 853-868.

Haidt, J. \& Hersh, M.A. (2001). Sexual morality: The cultures and emotions of conservatives and liberals. Journal of Applied Social Psychology, 31, 191-221.

Haidt, J. (2001). The emotional dog and its rational tail: A social intuitionist approach to moral judgment. Psychological Review, 4, 814-834.

Haidt, J. (2003). The moral emotions. In R.J Davidson, K.R. Scherer, \& H. H. Goldsmith, Handbook of affective sciences (pp. 852-870). Oxford: Oxford University Press. 
Hejmadi, A., Rozin, P., \& Siegal, M. (2004). Once in Contact, Always in Contact: Contagious Essence and Conceptions of Purification in American and Hindu Indian Children. Developmental Psychology, 40, 467-476.

Izard, C. E. (1977). Anger, disgust, and contempt and their relationship to hostility and aggression. London, Plenum Press.

Johnson-Laird, P. N., \& Oatley, K. (1989). The language of emotions. Cognition \& Emotion, 1, 29-50.

Keltner, D., \& Haidt, J. (2001). Social functions of emotions. In T. Mayne \& G.A. Bonanno (Eds.), Emotions: Current issues and future directions (pp. 192-213). New York: Guilford Press.

Kenny, D. A., Kashy, D. A., \& Bolger, N. (1998). Data analysis in social psychology. In D. Gilbert, S. T. Fiske, \& G. Lindzey (Eds.), The handbook of social psychology (4th ed., Vol. 1, pp. 223-265). New York: McGraw-Hill.

Kohlberg, L. (1969). Stage and sequence: The cognitive-developmental approach to socialization. In D. A. Goslin (Ed.), Handbook of socialization theory and research (pp. 151-235). New York: Academic Press.

Lerner, J. S., \& Keltner, D. (2001). Fear, anger, and risk. Journal of Personality and Social Psychology, 81, 146-159.

Levenson, R. W., Ekman, P. \& Friesen, W. V. (1990). Voluntary facial action generates emotion specific autonomic nervous system activity. Psychophysiology 27, 363-384 
Marzillier, S. L., \& Davey, G. C. L. (2004). The emotion profiling of disgust eliciting stimuli: Evidence of primary and complex disgust. Cognition and Emotion, 18, 313-336.

Nabi, R. L. (2002). The theoretical versus the lay meaning of disgust: Implications for emotion research. Cognition and Emotion 16, 695-703.

Olatunji, B.O. (2008). Disgust, scrupulosity and conservative attitudes about sex: Evidence for a mediational model of homophobia. Journal of Research in Personality, 42,1364-1369.

Ortony, A., Clore, G. L., \& Collins, A. (1988). The cognitive structure of emotions. Cambridge: Cambridge University Press.

Rozin, P., Haidt, J., \& McCauley, C.R. (1993).Disgust. In M. Lewis, \& J.M. Haviland (Eds.),Handbook of Emotions (pp.579-594). London: Guildford Press.

Rozin, P., Lowery, L., Imada, S., \& Haidt, J. (1999). The CAD hypothesis: A mapping between three moral emotions (contempt, anger, disgust) and three moral codes (community, autonomy, divinity). Journal of Personality and Social Psychology, 74: 574-586.

Rozin, P., Millman, L. \& Nemeroff, C. (1986). Operation of the laws of sympathetic magic in disgust and other domains. Journal of Personality and Social Psychology, 50, 703-712.

Russell, J. A., \& Fehr, B (1994). Fuzzy concepts in a fuzzy hierarchy: Varieties of anger.Journal of Personality and Social Psychology, 67, 186-205. 
Russell, P.S., \& Giner-Sorolla, R. (2009). Anger, not disgust, responds to intentionality. Unpublished Manuscript.

Simpson, J., Carter, S., Anthony, S.H., \& Overton, P.G. (2006). Is disgust a homogeneous emotion? Motivation and Emotion, 30 ,31-41.

Taylor, K. (2007). Disgust is a factor in extreme prejudice. British Journal of Social Psychology, 46, 597-617.

Tracy, J. L., \& Robins, R. W. (2008) The automaticity of emotion recognition. Emotion, 8), 81-95.

Weber, H. (2004). Explorations in the social construction of anger. Motivation and Emotion, 28, 197-219.

Wheatley, T., \& Haidt, J. (2005). Hypnotic disgust makes moral judgments more severe. Psychological Science, 16, 780-784. 
Social Justifications 34

Author Note

We wish to thank Jessica L. Tracy for sharing emotional expression photographs for use in this research. 
Social Justifications 35

Bodily Groups

Anger

Disgust
$5.25(2.26)$

$6.33(2.40)$
Non-bodily Groups

$5.32(2.17)$

$5.51(1.98)$

Table 1

Emotion Intensity Means by Group Type and Emotion for Experiment 2

Note. Standard deviations are in parentheses 
Social Justifications 36

\section{Figure Captions}

Figure 1. Reasons Means by Condition for Experiment 1

Figure 2. Reasons Means by Condition for Experiment 2

Figure 3. Reasons Means by Condition for Experiment 3

Figure 4. Type of Elaborated Reasons by Condition for Experiment 3 
Social Justifications 37

Figure 1

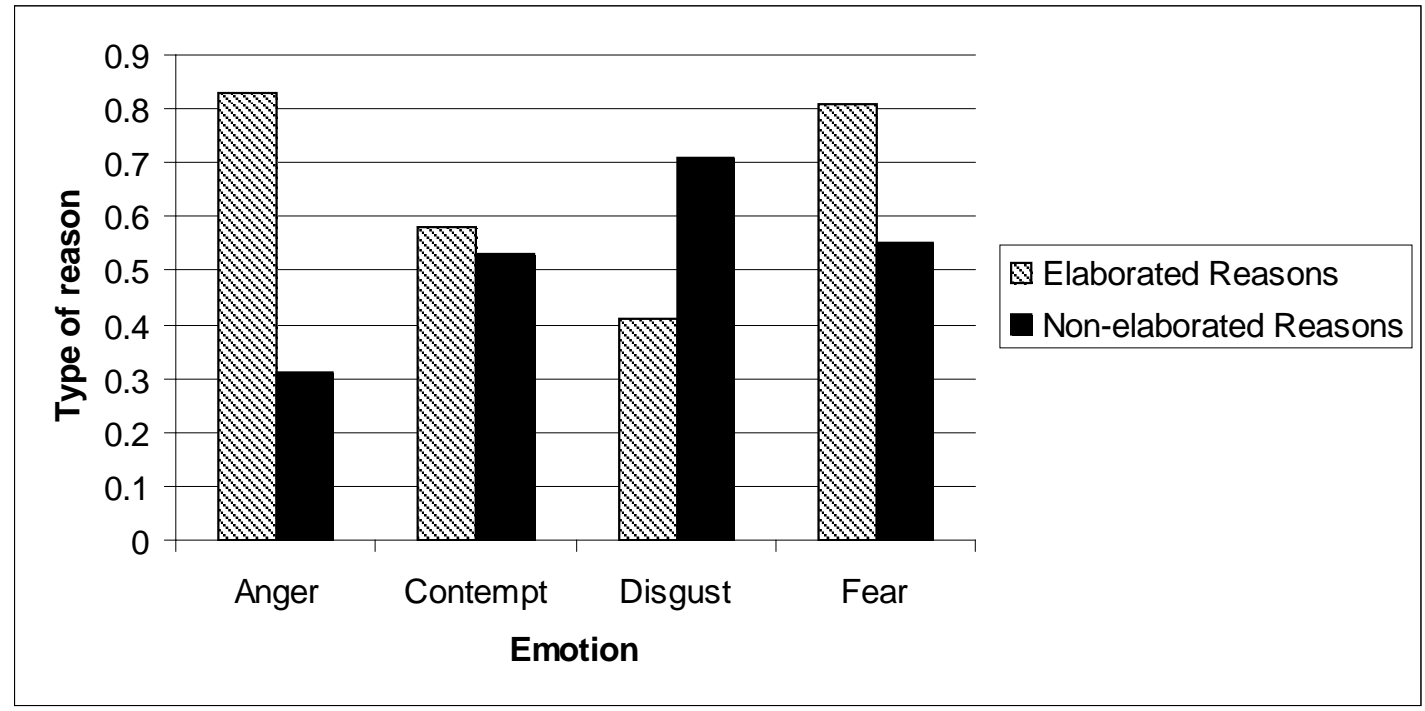


Social Justifications 38

Figure 2

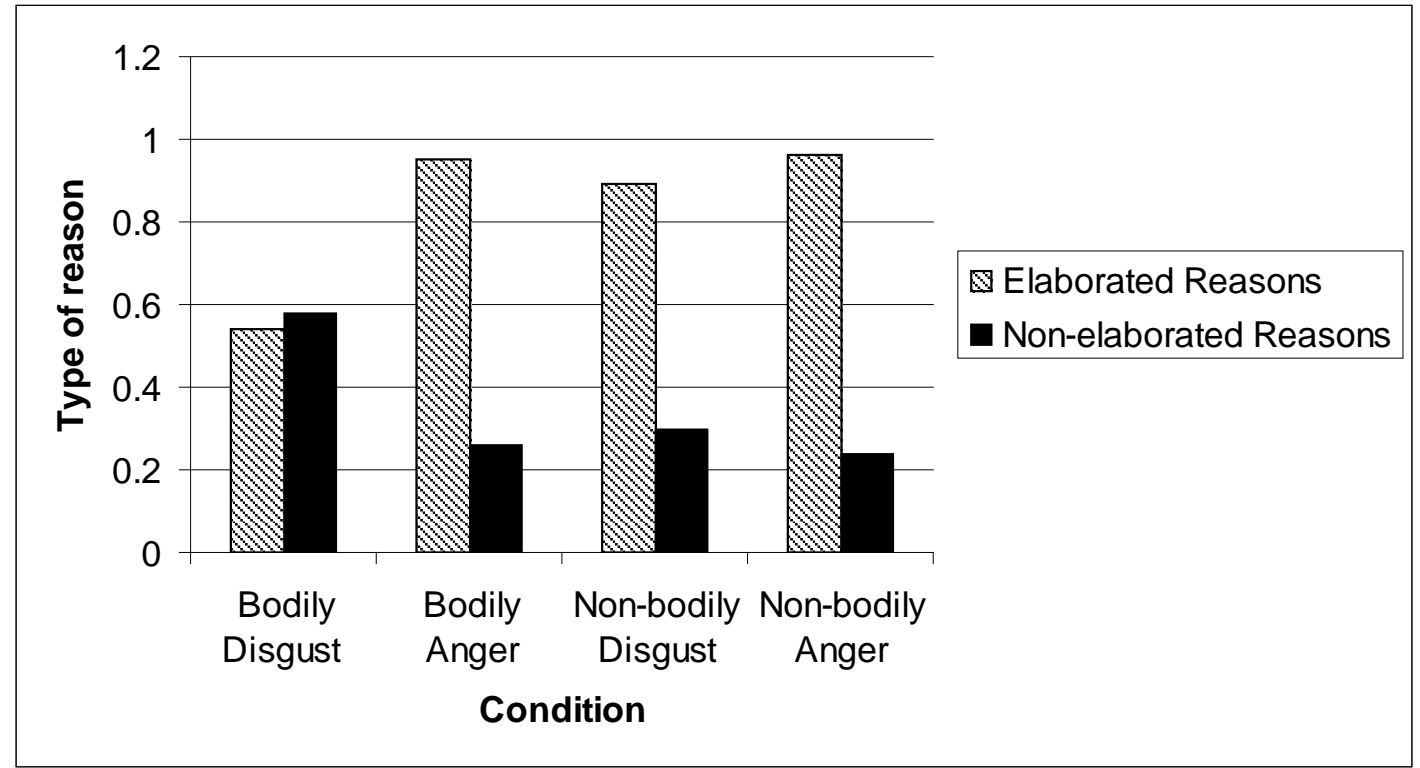


Social Justifications 39

Figure 3

Explanations Not Provided

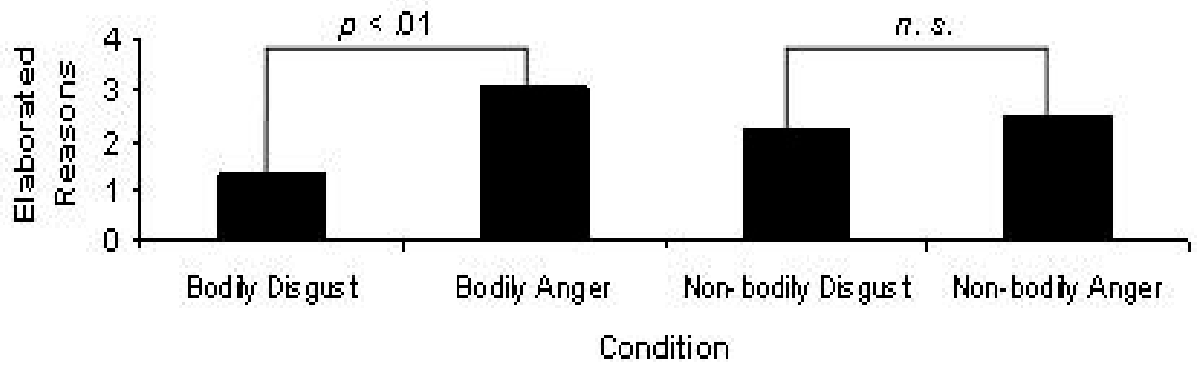

Eplanations Provided

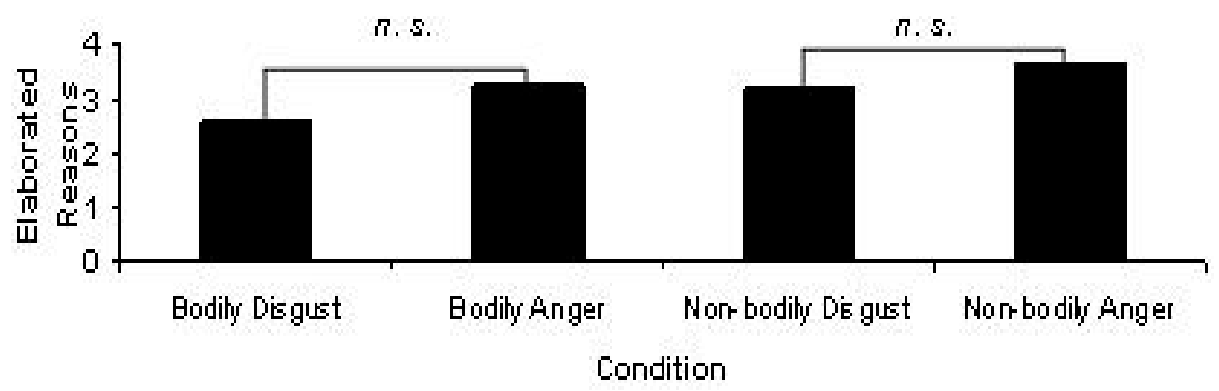


Social Justifications 40

Figure 4

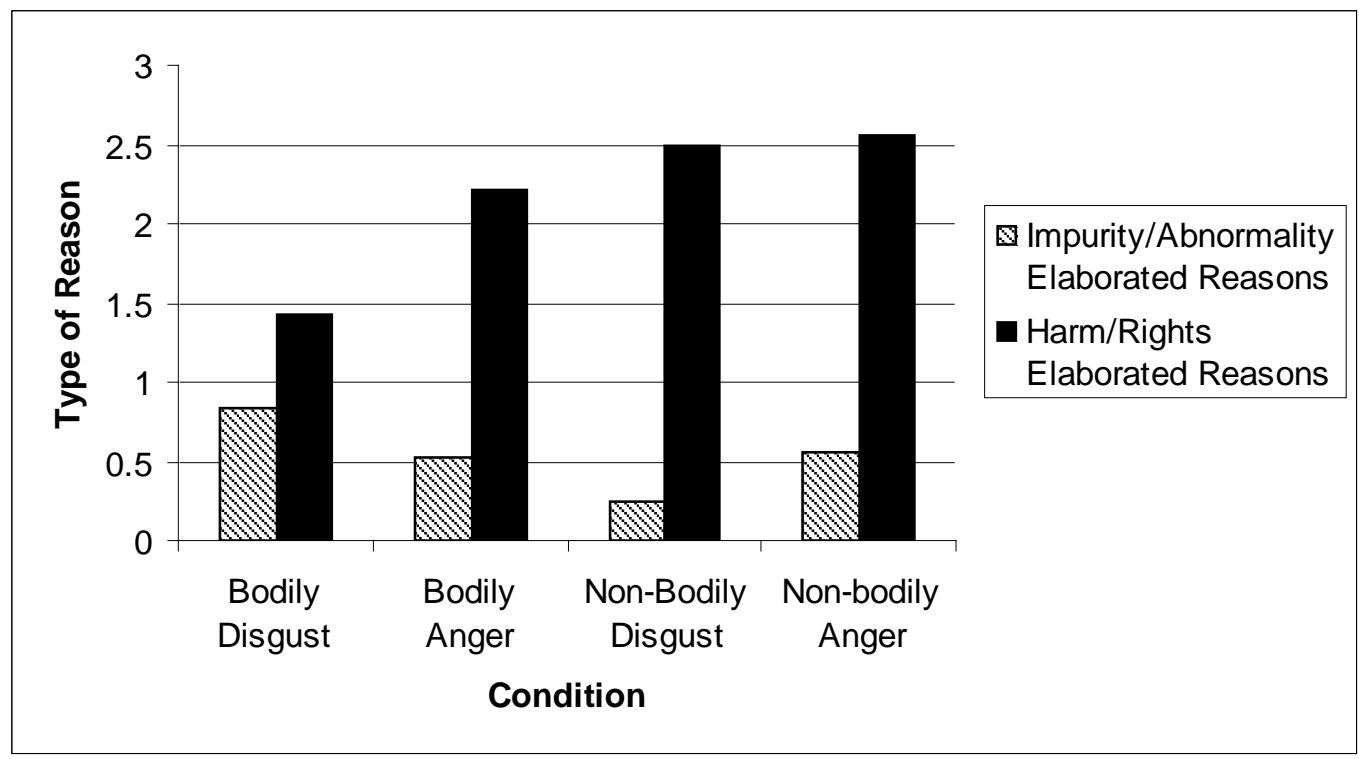


Social Justifications 41

\section{Appendix A}

\section{Instructions for Pre-test}

We have included neutral examples using the object "orange fizz" in place of a social group in order to provide an example on how to use the following scale.

1 - A simple, purely emotional or evaluative (good/bad) statement - no matter how many terms it uses to express this

Example: "I like orange fizz"; "Orange fizz is awful and bad”

3 - A statement that involves a simple judgment that is more specific than emotional or evaluative, but does not spell out any specific cause or consequence of the group or its behavior

Example: "Orange fizz is harmful”; "Orange fizz is not a normal drink"

5 - A statement that mentions a single, specific cause or consequence of the group in question or its behavior

Example: "Orange fizz puts innocent children at risk"; "Orange fizz is not a normal drink because it contains many artificial flavors"

7 - A statement that mentions multiple different causes or consequences

Example: "Orange fizz is harmful to children that drink it and makes them really hyper; therefore, it is bad for children" 
9 - A statement that not only mentions multiple causes or consequences but links them together

Example: "Orange fizz is harmful and makes children really hyper because of the large amount of sugar and artificial flavors that are contained in this drink"

Use the even numbers to indicate statements that, for you, fall in between two categories.

Appendix B

\section{General Instructions:}

Frequently in everyday life, we are asked why we feel certain emotions toward other persons and groups.

We may be asked about our emotions either because persons do not understand why we feel that way, or because they have had no prior contact with whatever is causing us to feel that emotion.

Therefore, within this study I would like you to take the role of someone who is trying to explain the reasons for feeling their emotion to other persons. You are trying to make the other person understand why you feel the way you do.

Within your explanation it is important that you achieve two goals: First, that you are able to explain your emotion as convincingly as possible, so that the other person will understand your reasons; second, to do this as economically as possible, using the minimum number of statements you think will convince the average fellow student. The responses you give will be compiled in order to create a prototypical argument for emotion explanation to other students. No one will be specifically linked to their answers; only a general argument will be created from all of the responses given within this study.

Specifically, I want you to explain the anger/ disgust you might feel toward:

- Group description presented

- Emotion face and emotion word presented

\section{Instructions for emotion explanations provided conditions:}

From this list please use the following statements to compile an argument that you would use to explain your anger/disgust toward this group. Within your response you can use as many or as few statements that you feel necessary, but not more than you need to convince others why you feel the way you do. Make sure when copying the statements that you copy them word for word. 
Instructions for emotion explanations not provided conditions:

Please provide a list of statements that you would use to explain your anger/disgust toward this group. Within your response you can use as many or as few statements that you feel necessary, but not more than you need to convince others why you feel the way you do. Please do not turn the page until you are done listing your statements. 